\title{
Towards a Wearable Wheelchair Monitor: Classification of push style based on inertial sensors at multiple upper limb locations
}

\author{
Roxana Ramirez Herrera \\ UCL Interaction Centre \\ University College London \\ London, UK \\ roxana.herrera.15@ucl.ac.uk
}

\author{
Behzad Momahed Heravi \\ Computer Science \\ University College London \\ London, UK \\ b.heravi@ucl.ac.uk
}

\author{
Giulia Barbareschi \\ UCL Interaction Centre \\ University College London \\ London, UK \\ giulia.babareschi.14@ucl.ac.uk
}

\author{
Tom Carlson \\ UCL Aspire CREATe \\ Catherine Holloway \\ UCL Interaction Centre \\ University College London University College London \\ Stanmore, UK \\ t.carlson@ucl.ac.uk \\ London, UK \\ c.holloway@ucl.ac.uk
}

\begin{abstract}
Measuring manual wheelchair activity by using wearable sensors is on the rise for rehabilitation and monitoring purposes. Stroke pattern is an important descriptor of the wheelchair user's quality of movement. This paper evaluates the capability of inertial sensors located at different upper limb locations plus wheel, to classify two types of stroke pattern for manual wheelchairs: semicircle and arc. Data was collected using bespoke inertial sensors with a wheelchair fixed to a treadmill. Classification was done with a linear SVM algorithm, and classification performance was computed for each sensor location in the upper limb, and then in combination with wheel sensor. For single sensors, forearm location had the highest accuracy $(96 \%)$ followed by hand $(93 \%)$ and arm $(90 \%)$. For combined sensor location with wheel, best accuracy came in combination with forearm. These results set the direction towards a wearable wheelchair monitor that can offer multiple on-body locations for increased usability.
\end{abstract}

Keywords - manual wheelchair; inertial sensor; push style; stroke pattern; wearable technology

\section{INTRODUCTION}

In 2011 the World Health Organization estimated that approximately $15 \%$ of the world population experiences some form of disability, with the number bound to rise as life expectancy increases [1]. Wheelchairs are one of the most common assistive technologies provided to enable independent mobility when people are unable, or struggle to walk. Wheelchair users account for approximately $10 \%$ of the whole disabled population [1]. Being able to move independently enables them to perform activities of daily living (ADLs), participate in social environments and better relate to other members of their community which, in turn has an important impact on their quality of life [2]. Furthermore, self-propelling a manual wheelchair also enables users to meet the suggested exercise requirements which aids in the prevention of cardiovascular and other non-communicable diseases (such as heart disease or diabetes) as well as to maintain musculoskeletal health [3]. However, wheelchair users do not often meet exercise requirements, with over $50 \%$ of the population exercising below the average requirements [4] .

The main limitations of wheelchair usage come from environmental factors such as lack of accessibility, but also from inexperience. Poorly trained users find it difficult to selfpropel and manage their daily surroundings effectively [4]. Lack of a good technique when self-propelling a manual wheelchair can lead to straining muscles or joints, leading to wear over time and permanent injury, which in turn can lead to permanent immobility [5].

The number of strokes when pushing, and the stroke pattern used when self-propelling are parameters that can describe the quality of the user's propelling technique, and have an influence on the likelihood of injury incurrence [6].

The arc and semi-circular patterns are two representative examples of over-rim and under-rim propulsive strokes, respectively (see Fig. 3) [7]. A semi-circular pushing style is usually recommended when covering long distances, particularly on level ground, as it allows for a smoother and more efficient movement that reduces musculoskeletal strain when compared to a fast arc pattern[8]. However, the arc pattern is more suited to uphill propulsion as it allows the user to increase cadence and better control the wheelchair [9]. It therefore becomes of therapeutic interest to identify and monitor the actual pushing style of each user in their everyday settings, as it provides an insight of the users' technique.

The use of inertial sensors has been examined in response to the rising interest in quantifying movement in wheelchair users for monitoring and rehabilitation purposes, due to their size, inexpensiveness and the fact that are not bound to a clinical setting [10]. For example, accelerometers have been used to measure basic parameters such as bouts of mobility by Sonemblum et al [11] and to record more complex tasks which can then be combined with classifying algorithms to distinguish between wheelchair activities [12] or recognise wheelchair destabilizing conditions [13]. Less attention has been given to measuring stroke patterns with wearable sensors. Only two studies have begun to explore the measurement of quality of movement. First, French et al studied 4 types of propulsion patterns using a wrist inertial sensor and classified using two algorithms ( K-Nearest neighbours, and Support Vector Machine) [14]. Second, Holloway et al classified two push styles (semicircle and arc) outdoors and indoors with only a 
wrist inertial sensor [15]. Neither study investigated the ideal location of sensor.

Currently, quantification of wheelchair activities through inertial sensors relies on placing them on the wheel, upper arm or wrist, with the last one being the most common body location [10][16].Varying the location of the sensor and the number of sensors used has been done with a practical approach, mainly with the purpose of increasing accuracy of measurements. For example, Hiremath et al have used inertial sensors on the wrist, upper arm and wheel to classify 10 different wheelchair activities in 45 participants, and to determine which sensor is a better predictor for physical activities [17]. They concluded that multimodal measurement increases the accuracy of activity detection. Garcia-Masso et al have also compared performance of classifiers using accelerometers placed on both wrists, chest and waist, for identification of physical activity in 10 categories. Their results showed that a combination of all sensors provided best performance [12].

Even though many of the current wearable systems tested in literature [18] have been created as a precursor for the development of a tool for provision of feedback or monitoring to the wheelchair user, none of the current studies have motivated their sensor placement by the user's preferences or needs.

Carrington et al [16][19] and Malu et al [20] have raised concerns about the lack of accessibility in wearable fitness trackers for manual wheelchair users. Carrington et al [16] interviewed 5 wheelchair athletes and 5 occupational therapists on their experience and expectations with activity tracking wearable technology. Their qualitative analysis identified that factors such as aesthetics, and device visibility and flexibility on their on-body location, are opportunity areas for tracking devices intended for wheelchair users and that when unmet, can limit their use. Malu et al [20] performed a study where 14 wheelchair users evaluated two off the shelf wearable trackers (Fitbit One ${ }^{\mathrm{TM}}$ and $\mathrm{Moov}^{\mathrm{TM}}$ ) and reported their experience and perceptions. One output of this research was to identify the usage limitations that arise from the trackers physical design and placement. Seven users chose to wear the Fitbit ${ }^{\mathrm{TM}}$, which is a clip-on device, on their clothing (such as sleeve) mentioning that the location chosen was mainly due to ease of use. When evaluating the Moov ${ }^{\mathrm{TM}}$, whose watch-like form factor is widely accepted on non-disabled users, concerns were raised by 9 of the disabled users, due to difficulty of putting on the device independently with some mentioning the interference of wrist worn devices when self-propelling. Moreover, when users were asked for design suggestions on trackers, the interest of embedding them in gloves became a recurrent theme.

In response to these findings, we performed a feasibility study to a) evaluate the possibility of using an inertial sensor at the back of the hand (instead of wrist), forearm and arm for classifying two representative pushing styles (arc or semicircular pattern); b) evaluate which of these on-body locations is better and c) which sensor from the inertial measurement unit (accelerometer and/or gyroscope provides more information on classification.

This study will expand on the work presented in [15] and will set the direction for further research towards a wearable wheelchair monitor able to identify the quality of the propulsion technique of a user, and that can be worn at the user's body preferable location.

\section{THE WEARABLE TRAINER : A PROPOSAL}

We propose that the wheelchair trainer becomes part of the everyday accessories of users, giving them a choice of locations to place the device according to their personal preferences and needs. The device can provide information that will work as a "trainer" of wheelchair skills for the user.

In this case, we propose using the ARCCS sensor (Accessible Routes from Crowdsourced Cloud Services) [21] for data collection. The ARCCS sensor is a bespoke sensing unit with triaxial accelerometer, gyroscope, magnetometer and pressure sensor with wireless capabilities. Its compact size allows it to easily incorporate in different form factors, as illustrated in Fig.1. Allowing the user to make a choice of where to locate the sensor rather than imposing the position might increase the willingness to wear the technology. Wheelchair performance metrics can then be uploaded to a mobile phone and provide real-time feedback or off-line description of their activity. The system could not only integrate all validated methods for describing wheelchair user's physical activity, but also a description of the quality of the movement through the type of pushing style.

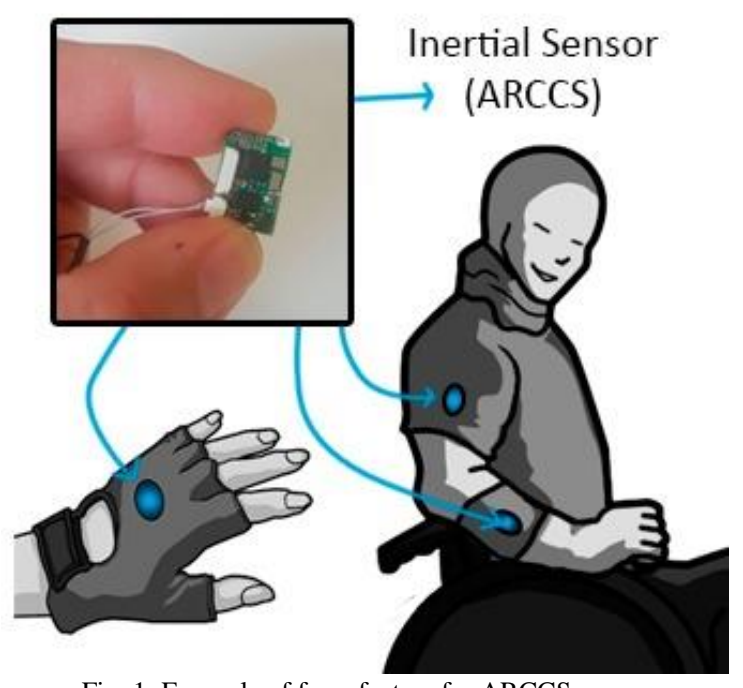

Fig. 1: Example of form factors for ARCCS sensor 


\section{CLASSIFYING PUSH STYLE FROM UPPER LIMB MOVEMENT}

\section{A. Data Collection}

To evaluate whether placing inertial sensors in different upper limb locations could be used to classify pushing patterns, and which location would be more suitable, we performed an inlaboratory feasibility study with a manual wheelchair fixed to a wheelchair treadmill. The study took place at the Mobility Laboratory at the Royal National Orthopaedic Hospital, in Stanmore, UK.

A physiotherapist trained on wheelchair provision and care was asked to perform several repetitions of level propulsions using one of two stroke patterns: arc, or semi-circular, at different self-selected speeds. The subject was wearing three ARCCS sensors (along with batteries) positioned at the top of the hand, the forearm, and upper arm as shown in Fig.2. In addition, one more sensor was attached on the wheelchair spokes. All four sensors logged triaxial acceleration and triaxial angular rate of rotation to a microSD card at a sampling rate of $100 \mathrm{~Hz}$. We used a CODA motion analysis system [22] to collect upper limb three-dimensional kinematic data that would serve as ground truth for classification of pushing style (Fig. 3). The body-worn sensors were safely attached to the subject with double sided tape, and markers for the CODA system were attached to hand and wrist. All trials were video recorded, and video analysis was used in combination with the CODA data to manually classify the type of stroke being performed, obtaining 48 examples for arc pattern and 39 for semi-circular pattern.

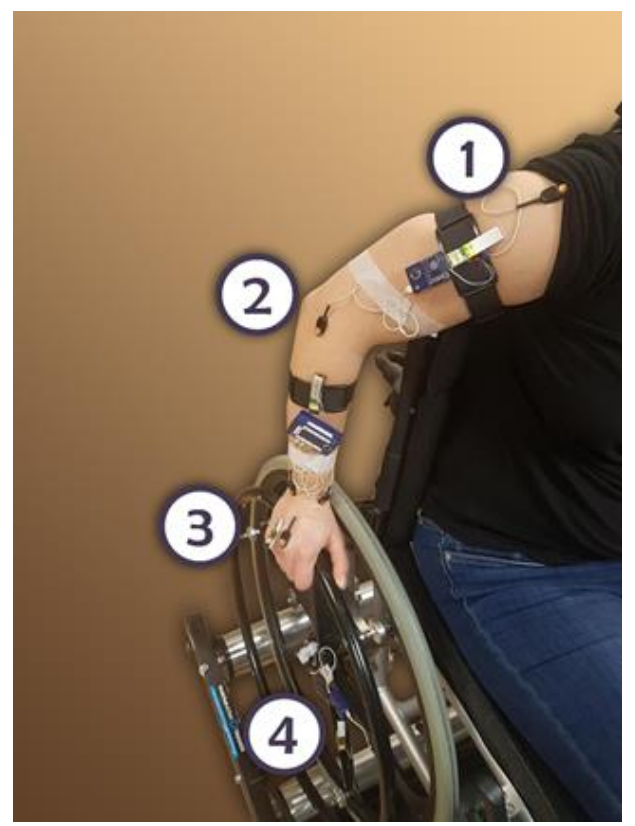

Fig. 2: Location of ARCCS sensors for experimental set up. $1=$ upper arm, $2=$ forearm, $3=$ hand, $4=$ wheel.

\section{B. Data Analysis}

Raw accelerometer and gyroscope data retrieved by the ARCCS sensors were plotted in Matlab 2017b (Fig. 4), along with the $3 \mathrm{D}$ position of the hand markers as collected with CODA. Orientation angles of each inertial sensor (yaw, pitch and roll) were calculated using the Madgwick filter [23]. We then divided the ARCCS data into non-overlapping sliding windows that covered each occurance of a wheelchair stroke. The window length was $1.25 \mathrm{~s}$, determined empirically from observing the ground truth plots. For each sample of push pattern we calculated a total of 18 features: mean and standard deviation of acceleration, angular rate and orientation angles across each of the 3 dimensional axes. We then used the features to train a linear Support Vector Machine (SVM) classifier, tested with a 10 -fold cross validation. We reported on precision, recall and accuracy (1-3) as metrics of classification performance for each sensor body location, and for 5 different feature groups: those related to i). accelerometer data, ii). gyroscope data, iii). gyroscope and accelerometer combined, iv). Euler angles and v). all together. All calulations were completed using custom-made code in Matlab2017b.

We then repeated the same procedure by combining predictors of each body-worn sensor with the ones from the wheel. No other combinations were evaluated as we considered that for better usability, the number of wearable sensors should be restricted to only one.

$$
\begin{aligned}
& \text { Precision = true positive } /(\text { true positive }+ \text { false positive }) \\
& \text { Recall = true positive } /(\text { true positive }+ \text { false negative }) \\
& \text { Accuracy = total correctly classified/ no. of cases }
\end{aligned}
$$

\section{Results}

The classifier performance metrics for single inertial sensors at locations 1, 2, 3 and 4 from Fig. 2 can be seen in Table I.

The activity classifiers trained with data collected from on-body ARCCS sensors presented a better accuracy relative to the ones positioned on the wheel. The highlighted cells in Table I indicate the highest accuracy for each body location. Overall, the sensor which presented the best accuracy was the one located in the forearm, with a $96.6 \%$ accuracy when trained with all 18 predictors. The second-best accuracy was found with the sensor placed on the hand, presenting a $92.0 \%$ accuracy when using acceleration-related only predictors, followed by upper arm classifier trained with all 18 features $(90.8 \%)$. Table II presents classification performance when the SVM was trained with data from each on-body inertial sensor combined with the wheel. In this case, forearm plus wheel presented the highest accuracy (97.7\% with all features), followed by arm plus wheel (88.5\% for gyroscope) and hand plus wheel (87.4 accuracy for both accelerometer and all features).

Addition of wheel features to hand location decreased performance for acceleration-related features but increased the 
gyroscope only performance. In the case of the wheel-forearm combination, performance increased in all options except for acceleration only ones. Combination of arm and wheel features decreased accuracy across all feature groups.
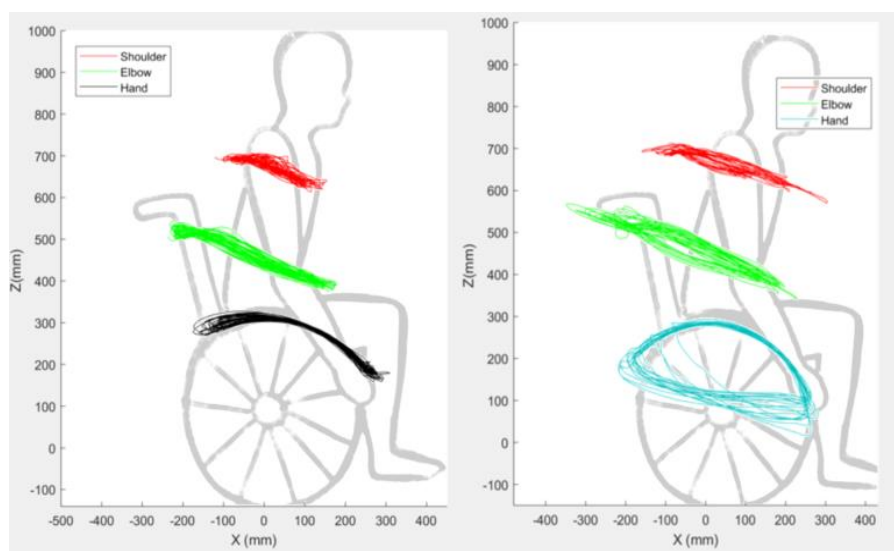

Fig. 3: Motion of CODA markers on shoulder, elbow and hand along X-Z axis (sagittal plane) as measured in the study. The graph on the left corresponds to an arc stroke pattern as seen from the hand trajectory presented in black. The graph on the right corresponds to a semi-circular stroke pattern (blue). Signals in green and red correspond to elbow and shoulder markers, respectively.
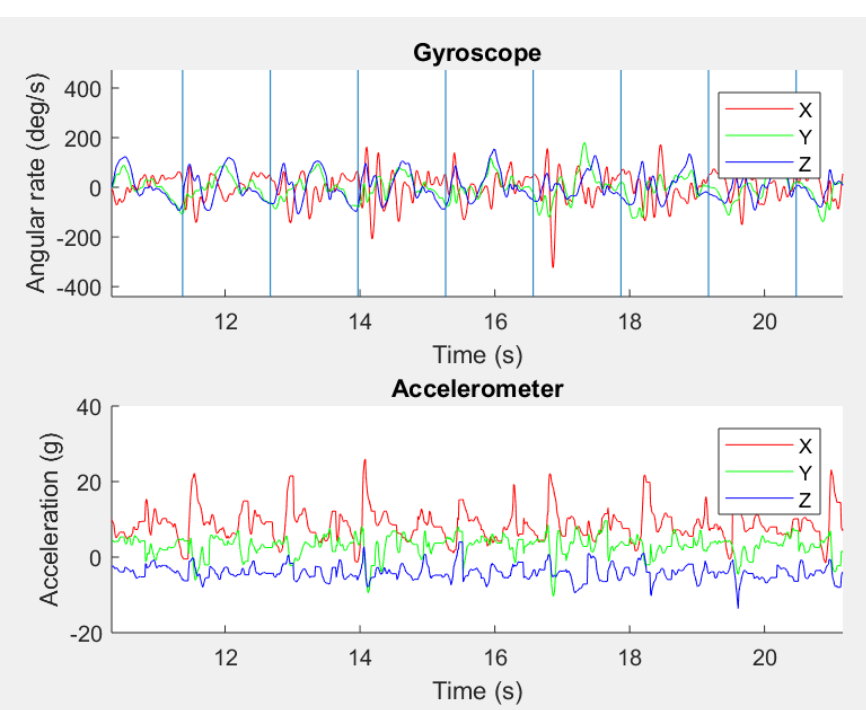

Fig. 4: Acceleration and angular rate across three orthogonal axes $(\mathrm{X}, \mathrm{Y}, \mathrm{Z})$ as collected by ARCCS sensor, from forearm location. Vertical blue lines separate each stroke window.

TABLE I: PERFORMANCE OF SVM FOR CLASSIFYING WHEELCHAIR PUSHING STYLE WITH INERTIAL SENSORS AT DIFFERENT BODY LOCATIONS

\begin{tabular}{|c|c|c|c|c|c|c|c|c|c|c|}
\hline \multirow{2}{*}{$\begin{array}{c}\text { Body } \\
\text { location }\end{array}$} & \multirow{2}{*}{$\begin{array}{c}\text { Feature } \\
\text { groups }\end{array}$} & \multicolumn{4}{|c|}{$\begin{array}{c}\text { Confusion Matrix (Semicircle }=\text { Class 1, } \\
\text { Arc }=\text { Class 0) }\end{array}$} & \multicolumn{2}{|c|}{ Semicircle (Class 1) } & \multicolumn{2}{|c|}{ Arc (Class 0) } & \multirow{2}{*}{$\begin{array}{c}\text { Overall } \\
\text { Accuracy } \\
{[\%]}\end{array}$} \\
\hline & & $\begin{array}{l}\text { true } 1, \\
\text { predict } 0\end{array}$ & $\begin{array}{l}\text { true } 1, \\
\text { predict } 1\end{array}$ & $\begin{array}{l}\text { true } 0, \\
\text { predict } 0\end{array}$ & $\begin{array}{l}\text { true } 0, \\
\text { predict } 1\end{array}$ & Precision & Recall & Precision & Recall & \\
\hline \multirow{5}{*}{ 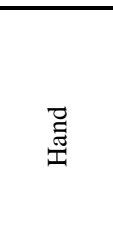 } & Accelerometer & 2 & 46 & 34 & 5 & 0.90 & 0.96 & 0.94 & 0.87 & 92.0 \\
\hline & Gyroscope & 9 & 39 & 31 & 8 & 0.83 & 0.81 & 0.78 & 0.79 & 80.5 \\
\hline & Acc + Gyro & 3 & 45 & 34 & 5 & 0.90 & 0.94 & 0.92 & 0.87 & 90.8 \\
\hline & Euler angles & 5 & 43 & 22 & 17 & 0.72 & 0.90 & 0.81 & 0.56 & 74.7 \\
\hline & All features & 1 & 47 & 29 & 10 & 0.82 & 0.98 & 0.97 & 0.74 & 87.4 \\
\hline \multirow{5}{*}{ 具 } & Accelerometer & 8 & 40 & 22 & 17 & 0.70 & 0.83 & 0.73 & $\overline{0.56}$ & 71.3 \\
\hline & Gyroscope & 3 & 45 & 32 & 7 & 0.87 & 0.94 & 0.91 & 0.82 & 88.5 \\
\hline & Acc + Gyro & 3 & 45 & 31 & 8 & 0.85 & 0.94 & 0.91 & 0.79 & 87.4 \\
\hline & Euler angles & 8 & 40 & 30 & 9 & 0.82 & 0.83 & 0.79 & 0.77 & 80.5 \\
\hline & All features & 3 & 45 & 34 & 5 & 0.90 & 0.94 & 0.92 & 0.87 & 90.8 \\
\hline \multirow{5}{*}{ 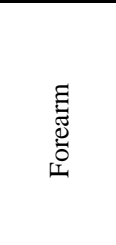 } & Accelerometer & 4 & 44 & 37 & 2 & 0.96 & 0.92 & 0.90 & 0.95 & 93.1 \\
\hline & Gyroscope & 8 & 40 & 23 & 16 & 0.71 & 0.83 & 0.74 & 0.59 & 72.4 \\
\hline & Acc + Gyro & 4 & 44 & 35 & 4 & 0.92 & 0.92 & 0.90 & 0.90 & 90.8 \\
\hline & Euler angles & 0 & 48 & 35 & 4 & 0.92 & 1.00 & 1.00 & 0.90 & 95.4 \\
\hline & All features & 0 & 48 & 36 & 3 & 0.94 & 1.00 & 1.00 & 0.92 & 96.6 \\
\hline \multirow{5}{*}{$\sum_{\bar{Z}}^{\bar{\Xi}}$} & Accelerometer & 7 & 41 & 24 & 15 & 0.73 & 0.85 & 0.77 & 0.62 & 74.7 \\
\hline & Gyroscope & 16 & 32 & 31 & 8 & 0.80 & 0.67 & 0.66 & 0.79 & 72.4 \\
\hline & Acc + Gyro & 12 & 36 & 31 & 8 & 0.82 & 0.75 & 0.72 & 0.79 & 77.0 \\
\hline & Euler angles & 14 & 34 & 14 & 25 & 0.58 & 0.71 & 0.50 & 0.36 & 55.2 \\
\hline & All features & 15 & 33 & 30 & 9 & 0.79 & 0.69 & 0.67 & 0.77 & 72.4 \\
\hline
\end{tabular}




\section{DISCUSSION}

This feasibility study showed it is possible to classify two stroke modalities: semicircle and arc, by placing inertial sensors in three locations in the upper limb different to the wrist, along with a linear SVM classifier trained using predictors extracted from basic kinematic variables. The forearm, followed by the hand, appear to be the most suitable locations for wearable inertial units, with accuracies for semicircle identification, comparable to the ones from [14] ( between $70-100 \%$ ) and slightly below the accuracy reported by [15] (98\%) both using wrist worn sensors. This latter's difference in accuracy could be attributed to our smaller sample size which can lead to relatively wide confidence intervals when comparing to those studies.

It is also observed that the most useful sensor features are relative to the sensor placement. In the hand and wheel placements, the accelerometer gives the most information, while in the upper arm and forearm positions, the gyroscope and Euler angles are the dominant factors.

Better performance of the forearm sensor can be attributed to the pushing stroke motion being aligned to the sagittal plane, showing a clearer distinction between motion axial components which allows for a clearer classification of movement. Even though the hand motion is clearly describing the semi-circular or arc trajectory, it has a lower accuracy compared to forearm.

TABLE II: PERFORMANCE OF SVM FOR CLASSIFYING WHEELCHAIR PUSHING STYLE WITH INERTIAL SENSORS AT DIFFERENT LOCATIONS ON BODY+ WHEEL

\begin{tabular}{|c|c|c|c|c|c|c|}
\hline \multirow{2}{*}{$\begin{array}{c}\text { Body } \\
\text { location }\end{array}$} & \multirow[b]{2}{*}{ Features } & \multicolumn{2}{|c|}{$\begin{array}{c}\text { Semicircle } \\
\text { (Class 1) }\end{array}$} & \multicolumn{2}{|c|}{ Arc (Class 0) } & \multirow{2}{*}{$\begin{array}{c}\text { Accurac } \\
\mathbf{y}[\%]\end{array}$} \\
\hline & & $\begin{array}{l}\text { Precision } \\
\end{array}$ & Recall & Precision & Recall & \\
\hline \multirow{5}{*}{ 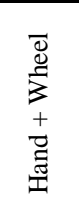 } & Accelerometer & 0.89 & 0.88 & 0.85 & 0.87 & 87.4 \\
\hline & Gyroscope & 0.82 & 0.94 & 0.91 & 0.74 & 85.1 \\
\hline & Acc + Gyro & 0.84 & 0.90 & 0.86 & 0.79 & 85.1 \\
\hline & $\begin{array}{c}\text { Euler } \\
\text { angles }\end{array}$ & 0.69 & 0.94 & 0.86 & 0.49 & 73.6 \\
\hline & All features & 0.85 & 0.94 & 0.91 & 0.79 & 87.4 \\
\hline \multirow{5}{*}{ 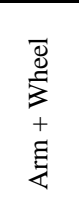 } & Accelerometer & 0.76 & 0.77 & 0.71 & 0.69 & 73.6 \\
\hline & Gyroscope & 0.87 & 0.94 & 0.91 & 0.82 & 88.5 \\
\hline & Acc + Gyro & 0.86 & 0.90 & 0.86 & 0.82 & 86.2 \\
\hline & $\begin{array}{c}\text { Euler } \\
\text { angles }\end{array}$ & 0.80 & 0.83 & 0.78 & 0.74 & 79.3 \\
\hline & All features & 0.84 & 0.79 & 0.76 & 0.82 & 80.5 \\
\hline \multirow{5}{*}{ 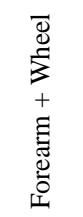 } & Accelerometer & 0.86 & 0.88 & 0.84 & 0.82 & 85.1 \\
\hline & Gyroscope & 0.86 & 0.90 & 0.86 & 0.82 & 86.2 \\
\hline & Acc + Gyro & 0.90 & 0.94 & 0.92 & 0.87 & 90.8 \\
\hline & $\begin{array}{c}\text { Euler } \\
\text { angles }\end{array}$ & 0.91 & 1.00 & 1.00 & 0.87 & 94.3 \\
\hline & All features & 0.96 & 1.00 & 1.00 & 0.95 & 97.7 \\
\hline
\end{tabular}

This could be explained due to the complexity of hand movement when performing a stroke; the orientation components of the sensor located at the back of the hand account for the translation of the hand, but also the extension and supination of the wrist, and radial and ulnar deviation. The complexity of this movement makes it difficult to use basic kinematic parameters. As for the case of arm, movement amplitude is smaller than the forearm and differentiation between patterns becomes less accurate.
Combining features from on-body sensors with the wheel did not improve accuracy for arm and hand placements, but it did provide the highest performance of them all (including precision and recall) when combined with forearm (97.7\%). Using inertial sensors on the wheel to measure basic activity metrics such as distance travelled or bouts of mobility has already been validated and tested in the wild [24]. Our results show that combining both sensors increases the quality and amount of information to describe wheelchair user's activity and performance.

From the results in Table 1, it can be observed that using only accelerometer data can potentially be enough to train algorithms for pushing style classification for on-body sensors. In the case of hand location, the best performance $(92.0 \%)$ was achieved when using only acceleration-derived features. As for the forearm inertial sensor, even though best performance $(96.6 \%)$ was found when using predictors from both accelerometer and gyroscope, acceleration-related predictors provided an accuracy of $93.0 \%$, which is still relatively high. The possibility of using only accelerometer data for feature extraction can have a positive impact in the battery life of the device.

One of the factors that influences the adoption of wearable devices is battery life and device size, which affect the device perceived ease of use [25]. As size restrictions mean battery capacity cannot be increased, diminishing energy consumption is the alternative approach to increase battery life. The ARCCS unit allows the flexibility of selecting type of data to be collected (i.e. acceleration only) and even changing sampling frequency, a feature unlikely to be modifiable in off-the-shelf devices and that also contributes to saving energy [21].

A limitation of this study is that for this preliminary stage, data collection took place in a controlled setting and with the wheelchair in a fixed position. Classifier performance needs to be tested with larger datasets from more subjects collected outdoors and indoors, over different surfaces and obstacles that account for the actual users' settings. In addition, the classifiers high accuracy was only for two distinguishable classes of pushing style: semi-circular and arc. Future versions of the system should consider classification of the other two over-rim and under-rim patterns identified in literature: single loop over propulsion (SLOP) and double-loop over propulsion (DLOP), which are variations of the arc and semi-circular pattern, respectively [26][7], as well as accounting for intermediate patterns.

Finally, as this method only intended to evaluate the trade-off between attributes used and location of sensors, it only used an SVM with basic predictors such as mean and standard deviation. As a design consideration towards the wearable trainer, using basic predictors would mean less computing time and better chances for providing real-time feedback to users. Future experiments will involve a thorough evaluation of a more complex feature space and evaluating other algorithms such as neural network classifiers in an attempt to learn the features instead of handcrafting them.

\section{CONCLUSION}

This work studied the feasibility of using inertial sensors for classifying push styles in self-propelled manual wheelchairs. 
Two common pushing styles (i) semi-circular and (ii) arc were considered and tested using empirical data on treadmill. Inertial sensors were placed in four different positions for a comparative study (arm, forearm, hand and wheel). A linear SVM was used to classify the two pushing styles.

Forearm position results showed the highest accuracy of classification (both recall and precision) for when accelerometer and gyroscope related attributes were used. Arm and Hand placements are similarly ranked second in terms of accuracy, and wheel placement comes last. Results also suggested the possibility of using only acceleration data when placed in the forearm and hand, for classification of pushing styles with reasonable accuracy (>90\%).

Future work will include evaluating the inertial sensor classification capabilities in outdoors and indoors settings with real wheelchair users, and evaluation of more complex feature space and algorithms to increase performance.

The results show great potential that a wearable inertial sensor such as the ARCCS can be used to classify push strokes and push styles. This research helps set the scene towards a wearable wheelchair monitor and possible trainer that allows the user to place the sensor at the most convenient body position, which would potentially improve its usability within the manual wheelchair population. As a sensor is only useful when used, this could help to increase activity levels in the manual wheelchair population if co-designed and developed with the targeted community.

\section{REFERENCES}

[1] World Health Organization, "World Report on Dissability," WHO Libr. Cat. Publ. Data, 2011.

[2] S. M. Hosseini, M. L. Oyster, R. L. Kirby, A. L. Harrington, and M. L. Boninger, "Manual Wheelchair Skills Capacity Predicts Quality of Life and Community Integration in Persons With Spinal Cord Injury," Arch. Phys. Med. Rehabil., vol. 93, no. 12, pp. 2237-2243, Dec. 2012.

[3] K. A. M. Ginis et al., "The development of evidence-informed physical activity guidelines for adults with spinal cord injury," Spinal Cord, vol. 49, no. 11, pp. 1088-1096, Nov. 2011.

[4] E. M. Smith, B. M. Sakakibara, and W. C. Miller, "A review of factors influencing participation in social and community activities for wheelchair users," Disabil. Rehabil. Assist. Technol., pp. 1-14, Dec. 2014.

[5] J. L. Mercer, M. Boninger, A. Koontz, D. Ren, T. Dyson-Hudson, and R. Cooper, "Shoulder joint kinetics and pathology in manual wheelchair users," Clin. Biomech., vol. 21, no. 8, pp. 781-789, Oct. 2006.

[6] Paralyzed Veterans of America Consortium for Spinal Cord Medicine, "Preservation of upper limb function following spinal cord injury: a clinical practice guideline for health-care professionals.," $J$. Spinal Cord Med., vol. 28, no. 5, pp. 434-70, 2005.

[7] A. M. Kwarciak, S. A. Sisto, M. Yarossi, R. Price, E. Komaroff, and M. L. Boninger, "Redefining the Manual Wheelchair Stroke Cycle: Identification and Impact of Nonpropulsive Pushrim Contact," Arch. Phys. Med. Rehabil., vol. 90, no. 1, pp. 20-26, Jan. 2009.

[8] M. L. Boninger, A. L. Souza, R. A. Cooper, S. G. Fitzgerald, A. M. Koontz, and B. T. Fay, "Propulsion patterns and pushrim biomechanics in manual wheelchair propulsion," Arch. Phys. Med. Rehabil., vol. 83, no. 5, pp. 718-723, May 2002.

[9] W. M. Richter, R. Rodriguez, K. R. Woods, and P. W. Axelson, "Stroke Pattern and Handrim Biomechanics for Level and Uphill Wheelchair Propulsion at Self-Selected Speeds," Arch. Phys. Med. Rehabil., vol. 88, no. 1, pp. 81-87, Jan. 2007.
[10] K. Tsang, S. V. Hiremath, T. M. Crytzer, B. E. Dicianno, and D. Ding, "Validity of activity monitors in wheelchair users: A systematic review," J. Rehabil. Res. Dev., vol. 53, no. 6, pp. 641-658, 2016.

[11] S. Eve Sonenblum, S. Sprigle, J. Caspall, and R. Lopez, "Validation of an accelerometer-based method to measure the use of manual wheelchairs," Med. Eng. Phys., vol. 34, pp. 781-786, 2012.

[12] X. García-Massó, P. Serra-Añó, L. M. Gonzalez, Y. Ye-Lin, G. PratsBoluda, and J. Garcia-Casado, "Identifying physical activity type in manual wheelchair users with spinal cord injury by means of accelerometers," Spinal Cord, vol. 53, no. 10, pp. 772-777, Oct. 2015.

[13] A. Crawford, K. Armstrong, K. Loparo, M. Audu, and R. Triolo, "Detecting destabilizing wheelchair conditions for maintaining seated posture," Disabil Rehabil Assist Technol, vol. 13, no. 2, pp. 178-185, 2018.

[14] B. French, A. Smailagic, D. Siewiorek, V. Ambur, and D. Tyamagundlu, "Classifying Wheelchair Propulsion Patterns with a Wrist Mounted Accelerometer," Proc. 3rd Int. ICST Conf. Body Area Networks, 2008.

[15] C. Holloway, B. Heravi, G. Barbareschi, S. Nicholson, and S. Hailes, "Street rehab: Linking accessibility and rehabilitation," in 2016 38th Annual International Conference of the IEEE Engineering in Medicine and Biology Society (EMBC), 2016, pp. 3167-3170.

[16] P. Carrington, K. Chang, H. Mentis, and A. Hurst, “'But, I don't take steps': Examining the Inaccessibility of Fitness Trackers for Wheelchair Athletes," Proc. 16th Int. ACM SIGACCESS Conf. Comput. Access. - ASSETS '15, no. To Appear, pp. 193-201, 2015.

[17] S. V Hiremath, S. S. Intille, A. Kelleher, R. A. Cooper, and D. Ding, "Detection of physical activities using a physical activity monitor system for wheelchair users," Med. Eng. Phys., vol. 37, pp. 68-76, 2015.

[18] D. Ding, S. Ayubi, S. Hiremath, and B. Parmanto, "Physical activity monitoring and sharing platform for manual wheelchair users.," 34th Annu. Int. Conf. IEEE Eng. Med. Biol. Soc. EMBS 2012, vol. 2012, pp. 5833-5836, 2012.

[19] P. Carrington, D. Ketter, and A. Hurst, "Understanding Fatigue and Stamina Management Opportunities and Challenges in Wheelchair Basketball," pp. 130-139, 2017.

[20] M. Malu and L. Findlater, "Toward Accessible Health and Fitness Tracking for People with Mobility Impairments," in Proceedings of the 10th EAI International Conference on Pervasive Computing Technologies for Healthcare, 2016, pp. 1-8.

[21] M. Gupta, C. Holloway, B. M. Heravi, and S. Hailes, "A comparison between smartphone sensors and bespoke sensor devices for wheelchair accessibility studies," 2015 IEEE 10th Int. Conf. Intell. Sensors, Sens. Networks Inf. Process. ISSNIP 2015, no. April, pp. 79, 2015.

[22] Codamotion, "3D Clinical Gait Systems - Reliable \& Easy." [Online]. Available: http://codamotion.com/index.php/technology/clinical-gait-analysis. [Accessed: 22-Apr-2018].

[23] S. O. H. Madgwick, A. J. L. Harrison, and R. Vaidyanathan, "Estimation of IMU and MARG orientation using a gradient descent algorithm," in 2011 IEEE International Conference on Rehabilitation Robotics, 2011, pp. 1-7.

[24] S. E. Sonenblum, S. Sprigle, and R. A. Lopez, "Manual wheelchair use: bouts of mobility in everyday life.," Rehabil. Res. Pract., vol. 2012, p. 753165, 2012.

[25] A. Adapa, F. F.-H. Nah, R. H. Hall, K. Siau, and S. N. Smith, "Factors Influencing the Adoption of Smart Wearable Devices," Int. J. Human-Computer Interact., vol. 34, no. 5, pp. 399-409, May 2018.

[26] A. M. Koontz, B. M. Roche, J. L. Collinger, R. A. Cooper, and M. L. Boninger, "Manual Wheelchair Propulsion Patterns on Natural Surfaces During Start-Up Propulsion," Arch. Phys. Med. Rehabil., vol. 90, no. 11, pp. 1916-1923, Nov. 2009. 